\title{
Numerical Simulation Analysis of Complex Variable Diameter Pipe Polishing by Solid-liquid Two-phase Abrasive Flow
}

\author{
Junye $\mathrm{Li}^{1}$, Xu Zhu${ }^{1}$, Hua Xiong ${ }^{2, *}$, Jinglei $\mathrm{Hu}^{1}$, Hui $\mathrm{Lu}^{1}$ and Baozuo Wang ${ }^{2}$ \\ ${ }^{1}$ College of Mechanical and Electric Engineering, Changchun University of Science and Technology, Changchun 130022, China \\ ${ }^{2}$ Changchun Aviation Hydraulic Pressure Control Corporation Limited. Changchun 130000, China \\ ${ }^{*}$ Corresponding author
}

\begin{abstract}
In order to explore the effect of abrasive flow on the variable diameter parts of the polishing effect, taking complex variable diameter pipe parts as the research object, the numerical simulation research on the machining process of abrasive particle flow polishing complicated diameter pipe parts was carried out, under the condition of different inlet pressure, distribution of dynamic pressure, velocity and turbulent viscosity of internal abrasive flow in complex variable diameter pipe parts. Through comparative analysis, the effectiveness of grinding and polishing complex variable diameter pipe parts is studied and analyzed, it can provide a theoretical basis for the continuous improvement of the abrasive flow super-finishing technology.
\end{abstract}

Keywords-abrasive flow polishing; complex variable diameter pipe; numerical simulation

\section{INTRODUCTION}

Complex variable diameter pipe is widely used in aviation, aerospace and automobile manufacturing industries ${ }^{[1,2]}$. With the rapid development of the automotive industry, now the requirements of the automotive industry are also getting higher and higher. On the basis of guaranteeing the performance, in addition to reducing the weight of the automobile itself, as well as saving energy and reducing emission, saving resources.

In order to meet different industrial requirements and prolong its service life, the design of complex variable diameter pipe has attracted more and more attention and attention. The process performance requirements of complex variable diameter pipe and other precision parts are getting higher and higher, the emergence of abrasive flow polishing technology has successfully solved this problem. ${ }^{[4]}$

Abrasive flow processing principle is the use of abrasive flow in the redo of numerous cutting tools (self-sharpening), cutting the machined surface repeatedly with its hard and sharp edges and corners, so as to achieve a certain processing purposes. ${ }^{[5-7]}$ Abrasive flow polishing technology is a new polishing method in recent years, the abrasive flow is generally formed by mixing a viscoelastic liquid carrier with solid particles of a certain stiffness and strength. ${ }^{[8]}$ Under the influence of external pressure, solid particles suspended in a liquid medium repeatedly impact the inner surfaces of complex structural parts, in order to achieve the smooth formation of the workpiece surface, polishing and material removal and other processing, so as to obtain higher precision. ${ }^{[9,10]}$

\section{BOUNDARY CONDITION SETTING AND NUMERICAL Simulation ANALYSIS OF COMPLEX VARIABLE DiAMETER PIPE BY ABRASIVE FLOW POLISHING}

A. Boundary Condition Setting of Complex Variable Diameter Pipe by Abrasive Flow Polishing

1) Inlet boundary condition

Continuous phase: The inlet pressure is applied to the abrasive inlet, it is assumed that the grit flow at the entrance is turbulent, K-epsilon (2 eqn) model for turbulence model selection.

Discrete phase: The discrete phase is a volume of silicon carbide particles, the concentration of discrete phase silicon carbide particles also affects the abrasive flow ability, if the particle flow in the solid particle content is too high, the formation of turbulence may be affected, even blocked the workpiece runner, therefore, the volume fraction of discrete phase should be chosen reasonably. Based on a large number of theoretical and practical research foundation, set the silicon carbide particle volume fraction of 0.2 , select abrasive particle size of $200 \mathrm{~nm}$, processing temperature is $310 \mathrm{~K}$. The same pressure inlet conditions are also used, the initial pressure is the same as that of the fluid.

\section{2) Exit boundary condition}

According to the actual process of abrasive flow machining of nozzle, it is known that the outlet is directly connected with the outside, and the abrasive flow can flow freely from the nozzle, so the outlet boundary condition is set as free outlet (outflow).

\section{3) Wall boundary conditions}

Since the abrasive flow used in the experiment belongs to the viscous fluid, in viscous flow, the wall surface defaults to the non-slip boundary condition.

\section{B. Numerical Simulation Analysis of Complex Variable Diameter Pipe by Abrasive Flow Polishing}

After setting the initial conditions, we selected $3 \mathrm{MPa}, 4 \mathrm{MPa}$, $5 \mathrm{MPa}, 6 \mathrm{MPa}$ four kinds of initial pressure was simulated, the simulated results show that multi physical coupling of four different initial stress field under the condition of the image, as shown in Figure 2 are given different initial pressure under 
dynamic pressure field nephogram.

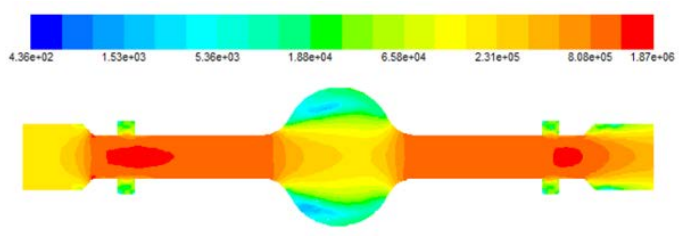

(a)The inlet pressure is $3 \mathrm{MPa}$

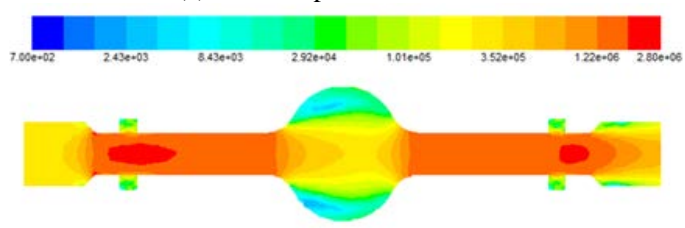

(b)The inlet pressure is $4 \mathrm{MPa}$



(c) The inlet pressure is $4 \mathrm{MPa}$

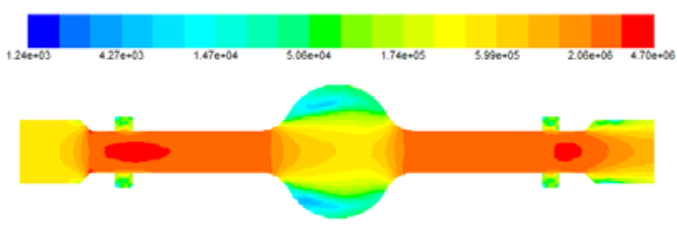

(d)The inlet pressure is $6 \mathrm{MPa}$

FIGURE I. DYNAMIC PRESSURE AT DIFFERENT INLET PRESSURE

From the dynamic pressure curves under different inlet pressures in Figure 2, it can be seen that under different inlet pressures, the trend of dynamic pressure distribution after abrasive flow processing is similar, at the entrance of the abrasive, the dynamic pressure is small, and the dynamic pressure increases when the abrasive enters the shrinkage hole at the entrance, this is because in the same flow rate, the diameter becomes smaller, the flow rate increases, the dynamic pressure becomes larger, so the polishing effect of small aperture is better than the polishing effect of large aperture, in the abrasive inflow entrance to the sudden change reaming, the middle of the sudden change in the hole and the outlet expansion reaming, the dynamic pressure is very small, which is due to the sudden increase in the diameter of the three reaming, and the abrasive only the right three to enter the collision Hole, and abrasive particles after the collision speed is relatively small, the entrance side of the mouth of the mutation expansion hole polishing effect is poor. With the increase of inlet pressure, the dynamic pressure tends to increase in the entire workpiece, and also shows a large increase rate in the small aperture, indicating that increasing the inlet pressure can increase the dynamic pressure and then enhance the grinding effect on the inner surface of the workpiece. In order to better grinding effect analysis inlet pressure on the workpiece, after the same initialization, the same four kinds of initial pressures are selected for numerical simulation. The simulation results of the multi-physics coupling field under four different initial pressures are obtained, as shown in Figure 3 are given different initial stress conditions of velocity field nephogram.

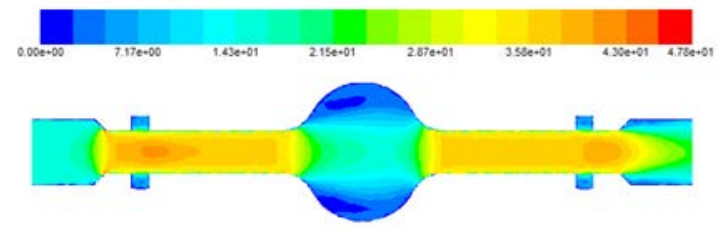

(a)The inlet pressure is $3 \mathrm{MPa}$

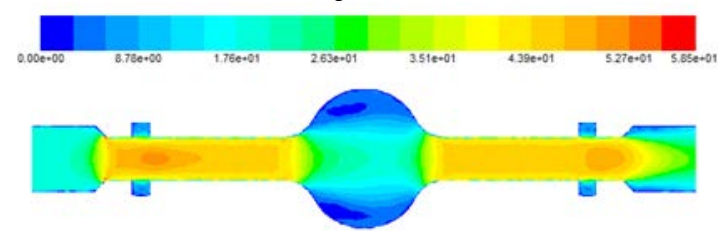

(b)The inlet pressure is $4 \mathrm{MPa}$

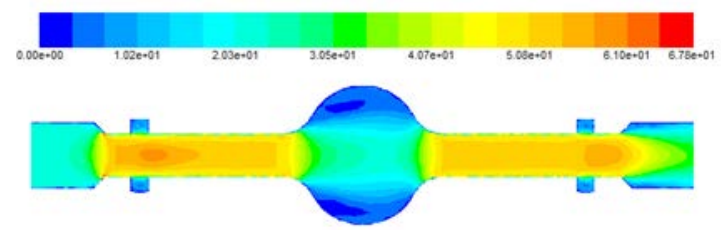

(c)The inlet pressure is 5MPa

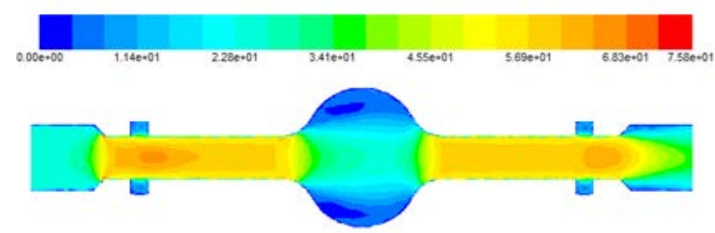

(d)The inlet pressure is 6MPa

FIGURE II. DIFFERENT INLET PRESSURE CONDITIONS SPEED CLOUD

As can be seen from the velocity profiles at different inlet pressures in Figure 3, as the inlet pressure continues to increase, most of the abrasive fluid is lost from the fluid as the abrasive flow enters the complex variable diameter pipe with a certain pressure. The fluid that produces the effect is less, due to the viscous effect of the abrasive medium, a boundary layer is formed in the near wall area of the machined surface and gradually extends along the flow direction, that is, the velocity of the fluid in the cavity close to the wall surface is lower than the flow velocity in the middle of the flow channel, This is due to the viscosity of the abrasive medium itself and friction caused by the wall and fluid flowing through the convex surface of the surface is hindered, leading to energy loss, resulting in speed reduction.

In order to better analyze the grinding effect of the inlet pressure on the workpiece, the same initialization, obtained under different initial pressure turbulent viscosity field cloud chart shown in Figure 4.

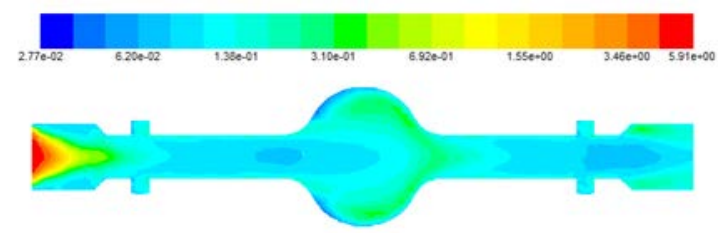

(a)The inlet pressure is $3 \mathrm{MPa}$ 


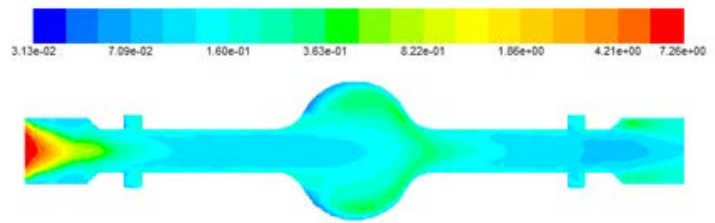

(b)The inlet pressure is $4 \mathrm{MPa}$

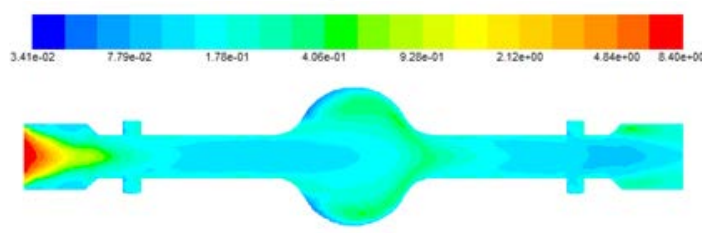

(c)The inlet pressure is $5 \mathrm{MPa}$

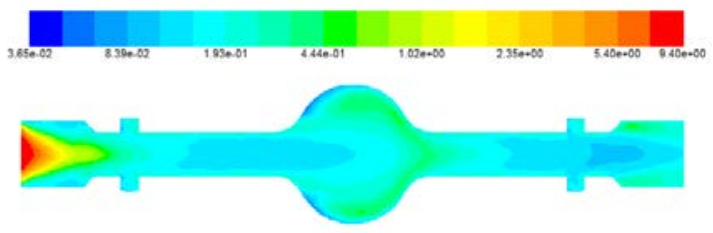

(d)The inlet pressure is $6 \mathrm{MPa}$

FIGURE III. TURBULENT VISCOSITY AT DIFFERENT INLET PRESSURES

From Fig. 4, the turbulent viscosity at different inlet pressures shows that when the inlet pressure increases, the turbulent viscosity tends to decrease and the fluidity of the fluid becomes better and better. Therefore, increasing the pressure is beneficial to increasing the particle velocity, the greater the abrasive velocity, the more violent the impact on the workpiece, thus the workpiece finishing and fillet.

\section{CONCLUSION}

Through the analysis of the dynamic pressure, velocity and turbulent viscosity in the process of abrasive flow polishing, it can be found that the abrasive flow has a significant effect on the rounding and deburring of the complex variable diameter pipe. The larger the inlet pressure, Polishing effect is better.

By changing the inlet pressure, different abrasive particle flow processing speeds can be obtained. Comparing different inlet pressures, it can be seen that the viscosity of the turbulent flow gradually decreases, the fluidity of the fluid is better, and the inlet pressure is increased, a better Abrasive grain speed, which can get better finishing effect.

Studying the dynamic pressure, velocity and turbulent viscosity in the flow field have some influence on the parameters of the abrasive flow, which can make the abrasive flow better and obtain the better processing result. After polishing the parts more in line with production requirements, improve the fatigue strength of parts, enhance the reliability of parts, extending its service life.

\section{ACKNOWLEDGMENT}

The authors would like to thank the national natural science foundation of china No. NSFC 51206011, Jilin province science and technology development program of Jilin province No.20160101270JC and No.20170204064GX, project of education department of Jilin province NO.2016386.

\section{REFERENCES}

[1] Shijian-Yuan, Lihui-Lang, Zhongren-Wang. Progress in research and application of tube hydroforming [J]. Journal of Harbin Institute of Technology,2000, (05):60-63.

[2] Shurong-Yu, Ding-Fan, Mingliang-Wu, Jinhui-Xiong, Jianhong-Chen. New hot working technologies for manufacture of space frame of aluminum alloys car bodies[J]. Hot working technology (cast forging version), 2006, (02):77-79.

[3] Baoliang-Zhang, Zhiming-Wang, Yi-Xie. Internal high pressure forming technology for automotive tapered reducing pipe fittings[J]. Die\&MouldIndustry, 2010,36(08):29-31.

[4] Junye Li, Lifeng Yang, Weina Liu, Fengyu-Sun. Experimental research into technology of abrasive flow machining non-linear tube runner[J], Advances in Mechanical Engineering, 2014, 752353,1-9.

[5] Shiming-Ji, Xihuang-Huang, Dapeng-Tan, Yunfeng-Tan.Gas-liquid-solid abrasive flow polishing and its process parameter optimization[J]. Optics and precision engineering, 2016,24(04):855-864.

[6] Jinglei-Hu, Junye-Li, Weihong-Zhao, Lili-Wei. Study on the Polishing of Variable Diameter Tube with Solid Liquid Two Phase Abrasive Flow[J], Journal of Changchun university of science and technology (Natural Science Edition), 2017,40(03):38-42.

[7] Weina-Liu, Zhijie-Cai, Yunfeng-LI, Chengzu-Ren. Numerical Simulation and Experiments of Abrasive Flow Polishing for Nozzle Micro-holes[J], China Mechanical Engineering, 2017,28(01):13-19+26.

[8] Junye-Li, Ying-Xu, Lifeng-Yang, Weina-Liu. Research on Abrasive Flow Machining Experiments of Non-linear Tubes[J], China Mechanical Engineering, 2014,25(13):1729-1734.

[9] Junye-Li, Jinglei-Hu, Kun-Dong Et al. Technological parameter optimization and quality effects on solid-liquid phase abrasive flow polishing[J], Optics and precision engineering, 2017,25(06):1534-1546.

[10] Junye-Li, Weina-Liu, Lifeng-Yang, Study of Abrasive Flow Machining Parameter Optimization Based on Taguchi Method[J], Journal of Computational and Theoretical Nanoscience,2013,10(12):2949-2954. 